\title{
How podcasts can benefit scientific communities
}

\author{
Daniel S. Quintana \\ University of Oslo
}

\author{
James A.J. Heathers \\ Northeastern University
}

\begin{abstract}
Podcasts are emerging as a popular forum for discussing scientific life. Here, we describe how podcasts can benefit scientific communities by disseminating career-specific information that is often unwritten and unavailable to those outside academic social knowledge networks. We also provide practical advice on how scientists can launch their own podcasts.
\end{abstract}

Keywords: science communication, scientific life, podcasts

Podcasts are digital audio files that are distributed over the internet. These audio files are typically spoken word and released in a regular episodic format. Podcasts emerged in the mid 2000's but took a decade to reach maturity, which can be attributed to several factors, including; (a) the increasing entrance of celebrities and public figures into podcasting, (b) the ubiquity of smartphones, (c) the first breakout successes (e.g., the Serial investigative journalism podcast series [1], which has been downloaded $\sim 300$ million times), and (d) the decreasing cost of entry for recording and distributing podcasts. Despite this rise in popularity, science podcasts are often overlooked as a means of consuming and communicating science. In this article, we will briefly describe the benefits of podcasts, drawing from our experience as co-hosts of Everything Hertz (everythinghertz.com), which is a podcast on scientific life and research methodology in the biobehavioral sciences that has been downloaded over half a million times.

\section{The benefits of podcasts for scientific communities}

Long working hours are the norm in the biological sciences. A global 2019 survey of more than 6000 graduate students revealed that $76 \%$ of the sample worked 41 hours a week or more [2]. It can be difficult keeping

Daniel S. Quintana, Norwegian Center for Mental Disorders Research (NORMENT), University of Oslo; James A.J. Heathers, Bouve College of Health Sciences, Northeastern University, Boston, USA. Work in the laboratory of DSQ is supported by a Novo Nordisk Foundation Excellence Grant (NNF16OC0019856). The authors would like to thank the listeners of the Everything Hertz podcast, some of whom provided feedback on an earlier version of this manuscript. Correspondence concerning this paper should be addressed to Daniel S. Quintana, Norwegian Center for Mental Disorders Research (NORMENT). Email: daniel.quintana@medisin.uio.no up-to-date with academic discussions with these demanding work schedules. A considerable advantage of audio podcasts is that they do not require visual attention, which means they can be heard while doing other tasks, such as commuting, driving, exercise, and home chores [3]. Anecdotally, we have been told by several listeners that they use our podcast episodes to accompany monotonous laboratory tasks, such as experiment preparation or manual data entry.

Audio communication is a popular type of asynchronous education delivery system [4]. Students rate podcasts as a more effective revision medium than textbooks [5], which can reduce the anxiety associated with high course loads [6]. Some scientific journals, such as Neuropsychopharmacology, produce podcasts that feature interviews of authors from recently published papers (nature.com/npp/podcast). This type of "behind the scenes" discussion provides the kind of nuance that is not possible with conventional academic papers, and is analogous to the casual conversations one may have with other scholars at conferences. Outside of one's own department, these types of conversations may be restricted to those with the resources and capability to attend academic conferences, but with a podcast, these insightful conversations can be made available for anyone to hear.

Most science podcasts are about science, focused on scientific topics or scientific output. While this podcast format serves an important role, very few science podcasts focus on being a scientist, the experience of scientific work, or scientific culture, in the biobehavioral sciences. It was with this in mind that we launched Everything Hertz in early 2016, with several other podcasts later joining this space, such as ReproducibiliTea 


\section{Box 1. Podcast hardware and software}

Podcasting is relatively inexpensive. The most important item of equipment is a dedicated microphone, typically connected to a workstation via USB. A microphone with a headphone output is preferable as these let you hear your own voice, which helps you adjust your microphone technique (e.g., the distance between your mouth and the microphone). Your headphones are also used to hear your co-host and/or guest, and prevent your own microphone from picking up their voices, which can be difficult to remove during editing. Audio can be recorded using free software, such as Audacity (audacityteam.org). Video conferencing software can be used when speaking remotely with co-hosts or guests, however, each participant should record their own audio track, which can be later edited together into a single MP3 file for distribution. Most video conferencing services also allow the video recording of conversations, which can be released in addition to audio podcasts. For a detailed guide with hardware and software recommendations for scientific podcasting, see osf.io/exu5h/.

(soundcloud.com/reproducibilitea), The Black Goat (theblackgoatpodcast.com), and Two Psychologists Four Beers (fourbeers.fireside.fm).

Between work demands, career disruptions, moving between short-term jobs, and emigration, academic life can be lonely. There is also the underappreciated matter of intellectual isolation, which researchers can experience when working in an area their department does not specialise in. Mental illness can also be a considerable issue for many graduate students [2], often exacerbated by the stress of poor working conditions and shrinking job prospects. The discussion facilitated by podcasts can act as a normalizing force for graduate students, which would otherwise not be accessible. Indeed, it has been remarked that, "...listening to their [the podcast host's] conversations I can kind of feel like I'm part of the sort of social group in a way" [7].

While the primary scientific activity of performing research is communicated via scientific journals, there are two types of career-specific information which complement scientific research that are rarely written down as they are typically communicated in face-toface settings. The first is academic social knowledge, which includes what grant schemes are available, how to navigate difficult situations, and when new positions become available. Members of academic social knowledge networks have a distinct career advantage, and it can be difficult for early career researchers to break into these circles, especially those who are intellectually isolated in their departments. Despite the individual nature of consuming podcasts, podcast listeners still feel like they are "part of an ongoing conversation or story that was unfolding, sometimes just for them" [7]. The second type of seldom communicated academic information is the unwritten rules of academia, such as the idiosyncrasies of the academic publishing system. Discussions on scientific podcasts can provide valuable career-specific information for early career researchers, especially first-generation scholars. A podcast can be used to ameliorate this social boundary.

\section{Podcasts are accessible}

The majority of public academic communication is locked behind journal paywalls or disseminated at conferences that require registration fees and resources for travel. In contrast, podcasts are almost exclusively open-access, as podcasters seek to have their work achieve maximum impact. Through podcast directories (e.g., Apple podcasts, Google podcasts), they are available instantly, free, and in perpetuity on a variety of platforms, and are both downloadable and streamable. The open format of RSS feeds, which are used to distribute episodes, means that podcasts are not locked to specific software applications or online platforms.

The "Matthew Effect", in which successful scientists are more likely to have additional successes, is a considerable issue in science [8]. Traditionally, only established academics get the opportunity to communicate science, as broadcasting opportunities are limited when working with conventional media organizations. However, podcasts can circumvent this issue by providing an accessible format that can provide a voice for early career researchers and underrepresented minority groups, who would otherwise not have such opportunities. 


\section{Box 2. Editing podcast audio}

There are several audio characteristics commonly found in raw vocal recordings that can be edited to help provide a more enjoyable listening experience. Audio editing can be fully-automated in Auphonic (auphonic.com), which provides two hours of free audio processing per month. Auphonic removes background hums (e.g., air-conditioning units) and most mouth "smacking" noises (caused by saliva), which can be irritating to listeners. Additionally, Auphonic normalises the volume of your podcast both within your episode so that some sections or speakers are not louder than others, but also between podcasts to the industry standard level. While the results of this automated audio processing are generally good, we recommend double-checking the processed recording to confirm that no errors were introduced. Other software packages can provide fine-grained semi-automated audio editing (e.g., iZotope RX), which can additionally remove plosives (windlike sounds that are often produced when speaking the letters "b" and "p") and harsh hisses that can be produced when pronouncing the letter "s". There are several free software options for editing together your audio clips and exporting your file as an MP3, such as Audacity and GarageBand. For a detailed guide on audio editing, including "before and after" audio examples, see osf.io/exu5h/.

\section{Sustainability}

While the openness of the podcast medium brings considerable benefits, one limitation is the costs associated with distributing podcasts. Depending on the available features, podcast hosting costs USD \$10-20 per month. Many podcasters pay for hosting out of their own pockets if they cannot secure institutional support. Alternatively, hosting services are beginning to emerge that provide free podcast hosting (e.g., anchor.fm/). These services raise revenue by taking a cut of any advertising that the podcast chooses to include in their show. The long-term sustainability of this revenue model is unclear; however, the open nature of the podcast format makes switching podcast hosting services relatively easy, without interrupting subscriptions. An alternative to podcast advertising is to collect financial support from the audience, using a platform like Patreon (patreon.com). These financial support platforms collect a monthly payment from listeners on behalf of podcasters, taking a small cut themselves. Financial supporters are often provided bonuses (e.g., additional supporteronly episodes) in return for their support.

A second limitation with podcasting as a form of academic communication is longevity. For as long as you want the podcast available, you will need to continue paying hosting costs or rely on a free hosting service, whose future can be assumed to be more uncertain than a persistent academic digital object identifier (DOI). However, there are now venues for free long-term storage of audio files that can be linked with a DOI, such as Zenodo or Open Science Framework (for an example of archived podcast episodes, see osf.io/zj7y3/). While these services are useful for archiving audio files, they cannot be used as a podcast webfeed, which means listeners have no subscription or update function.

\section{Starting your own podcast}

Compared to other forms of digital media, audio podcasts can be created relatively inexpensively, mostly with equipment that scientists can already access, and free software (see Text Boxes 1 and 2). The resources and skills which underlie the gap between amateur and professional podcast production is considerably smaller than video. The one piece of equipment that people might not have on hand but should be acquired at a minimum when starting a podcast is a dedicated microphone. Microphones that are built into smartphones and desktops typically provide low-quality recordings, as they tend to pick up excessive background noise and do a poor job at faithfully reproducing the sound of a human voice due to their small size.

\section{Concluding remarks}

Academic hierarchy (institutional prestige, resource allocation, etc.) and its consequences (gift authorships, implicit authority, etc.) have long been derided as inequitable and unsustainable [9]. Podcasts represent a method of subverting this entirely - to speak directly to the scientific commons in a space which is entirely 


\section{QUINTANA AND HEATHERS}

open, unrestricted, and fully accessible. The only true price of admission is to be consistent and compelling.

\section{Conflict of interest statement}

The authors are co-hosts of the podcast Everything Hertz, which receives financial support from listeners and episode sponsors for podcast-related costs.

\section{References}

1 Berry, R. (2015) A Golden Age of Podcasting? Evaluating Serial in the Context of Podcast Histories. J. Radio Audio Media 22, 170-178

2 Woolston, C. (2019) PhDs: the tortuous truth. Nature 575, 403-406

3 Thoma, B. et al. (2020) An international, interprofessional investigation of the self-reported podcast listening habits of emergency clinicians: A METRIQ Study. Can. J. Emerg. Med. 22, 112-117

4 Mallin, M. et al. (2014) A Survey of the Current Utilization of Asynchronous Education Among Emergency Medicine Residents in the United States. Acad. Med. 89, 598-601

5 Evans, C. (2008) The effectiveness of m-learning in the form of podcast revision lectures in higher education. Comput. Educ. 50, 491-498

6 Pilarski, P.P. et al. (2008) From music to macromolecules: Using rich media/podcast lecture recordings to enhance the preclinical educational experience. Med. Teach. 30, 630-632

7 Heshmat, Y. et al. (2018) Quality 'Alone'Time through Conversations and Storytelling: Podcast Listening Behaviors and Routines., in Proceedings of the 44th Graphics Interface Conference, pp. 76-83

8 Petersen, A.M. et al. (2011) Quantitative and empirical demonstration of the Matthew effect in a study of career longevity. Proc. Natl. Acad. Sci. 108, 18-23

9 Clauset, A. et al. (2015) Systematic inequality and hierarchy in faculty hiring networks. Sci. Adv. 1, e1400005 